\title{
Bridging the divide in heart failure
}

\author{
British Society for Heart Failure 10th Annual Autumn Meeting
}

23-24 November, 2007, London, UK

\section{Martin R Cowie}

Chairman, British Society for Heart Failure, Imperial College London, SW3 6LY, UK

Tel.: +44 207351 8856; Fax: +44 207351 8148; E-mail: m.cowie@imperial.ac.uk

$\mathrm{H}$ eart failure largely affects the elderly, with an average age at first diagnosis of 75 years in most developed countries. $M$ any of these patients have comorbidity, such as chronic airways disease, diabetes, hypertension and renal dysfunction. Although the prognosis has improved dramatically in the past 10 years - largely owing to the more widespread introduction of drug therapies such as angiotensin-converting enzyme (ACE) inhibitors and $\beta$-blockers - recent surveys have shown that only a small minority of patients admitted to hospital with heart failure are seen by a specialist heart failure team, and less than $20 \%$ are followed up in a specialist clinic. The vast majority of patients in the UK are followed up in primary care, with some input of care from the elderly physicians. The use of drugs known to improve symptoms and prognosis remains less than ideal.

The first session of the meeting was a detailed discussion of the role of the primary care physician in the diagnosis and treatment of heart failure. Applying the guidance from NICE, with a normal ECG and normal plasma brain natriuretic peptide (BNP) measure ment making heart failure very unlikely, was often difficult - access to BN $P$ testing is still very limited in the UK. In addition, ECG interpretation is also of variable quality and, in the elderly, the ECG is rarely completely normal. Data from the Quality and 0 utcomes Framework suggest that the quality of diagnosis in primary care varies markedly, and this is supported by a recent $\mathrm{H}$ ealth $\mathrm{C}$ are $\mathrm{C}$ ommission survey that demonstrates marked variation in speed of access to diagnostic tools such as echocardiography.

General practitioners with a specialist interest (GPSI) in cardiovascular disease are playing an increasing role in the diagnosis and management of more complex cardiac problems, including heart failure. This is observed to be most effective where there are good links to the secondary care-based cardiologists. Evidence was presented to show the improvement in the timetodiagnose heart failure and better uptake of appropriate drug therapy in areas where a GPSI had been established.

Primary care physicians also play a key role in ensuring uptitration of medica tion, such as ACE inhibitors, angiotensin-receptor blockers and $\beta$-blockers. $M$ easuring blood pressure, pulse rate and renal function can easily be carried out in primary care, saving the patient a journey to the local hospital, but does require an interested primary-care physician and practice nurse. Increasingly, heart failure specialist nurses are working in the community, often funded by the British $\mathrm{H}$ eart Foundation or Primary Care Trusts. Evidence was presented demonstrating that this initiative has reduced inappropriate hospital admissions and is cost effective.

Multidisciplinary work is the best model of care, a point emphasized throughout the meeting. Increasingly, the competenceand knowledge of the healthcare professional is more important than where they work. H eart failure patients benefit from good access to medical and nursing expertise, and also to pharmacists, physiotherapists, social workers and those with palliative care skills. The roles of the different professionals are evolving rapidly, with many tasks previously undertaken by medical staff now devolved to specialist nurses, including uptitration of medication and examining blood tests.

Although a multidisciplinary approach can improve outcomes in patients with heart failure, particularly in an area where services have been poor, it was agreed that programs need to be tailored to the needs of the local area - one size does not fit all. Tailoring service to accomodate the needs of the patient, which change over time, is also key and requires good communication between professionals and between the patient, family and health and social services.

A recently completed randomized trial of a nurse-led intervention in The N etherlands (Coordinating Study Evaluating Outcomes of Advising and Counseling in $\mathrm{H}$ eart Failure [ $\mathrm{COACH}]$ ) was presented. There was no difference in the clinical outcome or healthcare utilization in patients randomized to two different levels of intensity of nurseled heart failure follow-up care (basic and intensive) versus usual care. 0 ver a follow-up period of 18 months, there was no difference in the incidence of all-cause mortality and heart failure readmission between the treatment groups. H owever, nurse-led counseling was associated with improved adherence to nonpharmacological treatment. Although somewhat at odds with earlier studies of nurseled intervention, the consensus at the meeting was that provided inpatient care is good, follow-up can be streamlined. An intensive followup regimen is probably not appropriate for all heart failure patients, and should be targeted at those most at risk of deterioration, such as those in $\mathrm{New}$ York 
Heart Association (NYHA) class III or IV, particularly with previous multiple hospital admissions.

All heart failure patients require palliation of symptoms and supportive care. Access to specialist palliative care skills has often been poor, but has improved in the UK in recent years, as it has done across Europe and North America. Barriers to implementation of good palliative care include the belief that it is only appropriate for a patient who is to die shortly, the difficulty of making an accurate prognosis and the challenge of telling patients about the choices and limitations of treatment. Although it is difficult to define standard referral criteria, it was suggested that patients with NYHA class III-IV heart failure, recurrent hospitalization for symptomatic decompensated heart failure (despite optimal medical therapy) and difficult physical or psychosocial issues would be most likely to benefit from this type of care. It was emphasized that all healthcare professionals should be able to provide a palliative approach to care, but will require access to more specialized skills from time to time.

The meeting concluded with brief presentations of recent clinical trials that will impact on heart failure practice. The Controlled Rosuvastatin Multinational Trial in H eart Failure (CORONA) study of rosuvastatin $10 \mathrm{mg}$ versus placebo in patients with heart failure and coronary artery disease [1], reported no difference in all-cause mortality, cardiovascular mortality or coronary events between the two arms, despite a profound reduction in serum cholesterol and C-reactive protein concentration. The suggestion was made that the natural history of the left ventricular systolic dysfunction overwhelmed the impact of the statin on the behavior of the coronary disease. Efficacy of Vasopressin Antagonism in $\mathrm{H}$ eart Failure O utcome Study with Tolvaptan (EVEREST), a study of the vasopressin V2-antagonist, tolvaptan, in patients admitted to hospital with heart failure [2], found that a minimum of 60 days oral treatment had no impact on mortality or cardiovascular death and subsequent heart failure hospitalizations, although improvement in breathlessness and diuresis was perhaps faster in the tolvaptan group during the first week of therapy compared with usual therapy. In STARS-BNP [3], a BN Pguided strategy halved the risk of death or hospitalization related to heart failure over a median of 15 months follow-up, compared with a clinical strategy alone. The mechanism of benefit appeared to be that clinicians were more likely to increase the dosage of ACE inhibitors and $\beta$-blockers in those patients where the target was to drive the plasma BNP concentration down to $100 \mathrm{pg} / \mathrm{ml}$.

Although no new therapy for heart failure has been discovered in the past year, the continued lack of general use at appropriate dose of therapies known to improve prognosis and quality of life, such as ACE inhibitors and $\beta$-blockers, continues to be a major challenge. U sing the workforce to improve the standards of care remains pivotal, based on good training, supervision and audit of what should be a multidisciplinary team spanning primary and secondary care.

\section{Information resources}

- Further information about the activities of the British Society for $\mathrm{H}$ eart Failure (BSH) and a copy of the BSH $N$ ewsletter, which includes summaries of all presentations made at this meeting, can be found at:

www.bsh.org.uk

- The 11th BSH Annual Autumn $M$ eeting will be held in London on Thursday 20th and Friday 21st N ovember 2008. Anyone interested in attending or who would like to become a $\mathrm{M}$ ember or Friend of the BSH should contact:

info@bsh.org.uk

Financial \& competing

interests disclosure

M R Cowie has provided consultancy advice to a number of companies with products marketed for the diagnosis or treatment of heart failure. The author has no other relevant affiliations or financial involvement with any organization or entity with a financial interest in or financial conflict with the subject matter or materials discussed in the manuscript apart from those disclosed.

No writing assistance was utilized in the production of this manuscript.

\section{Bibliography}

1. Kjekshus J, Apetrei E, Barrios V et al.; for the CORON A group: Rosuvastatin in older patients with systolic heart failure. N. Engl. J. M ed. 357, 2248-2261 (2007).

2. Konstam M A, Burnett J C, Gheorghiade M et al.: Effects of oral tolvaptan in patients hospitalised for worsening heart failure: the EVEREST outcome trial. JAM A 297, 1319-1331 (2007).

3. Jourdain $P$, Jondeau $G$, Funck $F$ et al.: Plasma brain natriuretic peptide-guided therapy to improve outcome in heart failure: the STARS-BN P multicentre study. J. Am. Coll. Cardiol. 49, 1733-1739 (2007). 